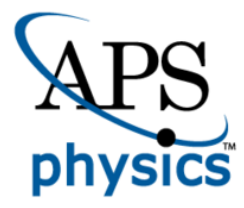

This is the accepted manuscript made available via CHORUS. The article has been published as:

\title{
Observation of the Unconventional Photon Blockade
}

H. J. Snijders, J. A. Frey, J. Norman, H. Flayac, V. Savona, A. C. Gossard, J. E. Bowers, M. P. van Exter, D. Bouwmeester, and W. Löffler

Phys. Rev. Lett. 121, 043601 - Published 23 July 2018

DOI: 10.1103/PhysRevLett.121.043601 


\title{
Observation of the Unconventional Photon Blockade.
}

\author{
H.J. Snijders, ${ }^{1}$ J. A. Frey,${ }^{2}$ J. Norman,${ }^{3}$ H. Flayac, ${ }^{4}$ V. Savona, ${ }^{4}$ A. C. \\ Gossard,$^{3}$ J. E. Bowers ${ }^{3}$ M. P. van Exter, ${ }^{1}$ D. Bouwmeester, ${ }^{1,2}$ and W. Löffler ${ }^{1}$ \\ ${ }^{1}$ Huygens-Kamerlingh Onnes Laboratory, Leiden University, \\ P.O. Box 9504, 2300 RA Leiden, The Netherlands \\ ${ }^{2}$ Department of Physics, University of California, Santa Barbara, California 93106, USA \\ ${ }^{3}$ Department of Electrical \&S Computer Engineering, \\ University of California, Santa Barbara, California 93106, USA \\ ${ }^{4}$ Institute of Physics iPHYS, École Polytechnique Fédérale de Lausanne EPFL, CH-1015 Lausanne, Switzerland
}

\begin{abstract}
We observe the unconventional photon blockade effect in quantum dot cavity QED, which, in contrast to conventional photon blockade, operates in the weak coupling regime. A single quantum dot transition is simultaneously coupled to two orthogonally polarized optical cavity modes, and by careful tuning of the input and output state of polarization, the unconventional photon blockade effect is observed. We find a minimum second-order correlation $g^{(2)}(0) \approx 0.37$ which corresponds to $g^{(2)}(0) \approx 0.005$ when corrected for detector jitter, and observe the expected polarization dependency and photon bunching and anti-bunching very close-by in parameter space, which indicates the abrupt change from phase to amplitude squeezing.
\end{abstract}

A two-level system strongly coupled to a cavity results in polaritonic dressed states with a photon-number dependent energy. This dressing gives rise to the photon blockade effect $[1,2]$ resulting in photon-number dependent transmission and reflection, enabling the transformation of incident coherent light into specific photon number states such as single photons. Single-photon sources are a crucial ingredient for various photonic quantum technologies ranging from quantum key distribution to optical quantum computing. Such sources are characterized by a vanishing second-order auto-correlation $g^{(2)}(0) \approx 0$ [3].

In the strong coupling regime, where the coupling between the two-level system and the cavity is larger than the cavity decay rate $(g>\kappa)$ [4], photon blockade has been demonstrated in atomic systems [5], quantum dots in photonic crystal cavities [6], and circuit QED [7, 8]. At the onset of the weak coupling regime $(g \approx \kappa)$, it has been shown that by detuning the dipole transition frequency with respect to the cavity resonance, photon blockade can still be observed [9]. However, moving further into the weak coupling regime $(g<\kappa)$ which is much easier to achieve $[10,11]$ (in particular if one aims for a small polarization mode splitting), conventional photon blockade is no longer possible because the energy gap between the polariton states vanishes. Nevertheless, also in the weak coupling regime, the two-level system enables photon number sensitivity, which has recently enabled highquality single photon sources using polarization postselection [12-14] or optimized cavity in-coupling [15][16].

In 2010, Liew and Savona introduced the concept of the unconventional photon blockade (UPB) [18, 19] which operates with arbitrarily weak non-linearities. It was first investigated for Kerr non-linearities [18, 20], then for $\chi^{(2)}$ non-linearities [21] and the Jaynes Cummings $[17,22]$ system which we focus on here. Both the con- (a) Photon blockade

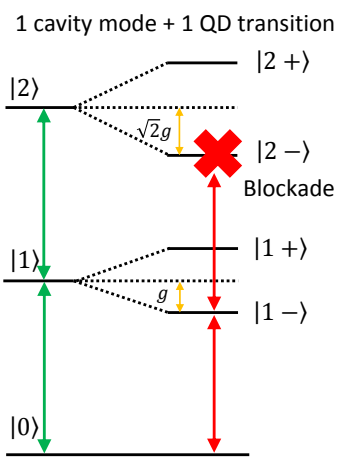

(b) Unconventional Photon blockade 2 cavity modes +1 QD transition

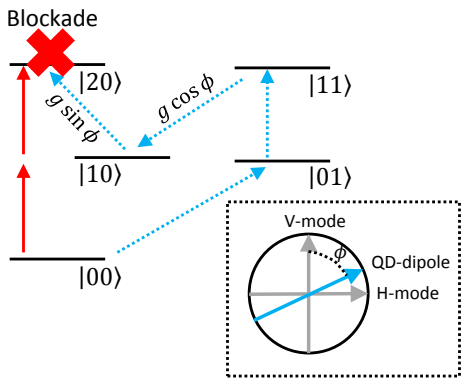

Figure 1. Removal of the 2-photon component in conventional photon blockade by the anharmonicity of the Jaynes Cummings ladder (a). In the unconventional photon blockade (b, adapted from [17]), two excitation pathways (red and blue arrows) destructively interfere. The state $|i j\rangle$ corresponds to $(i, j)$ photons in the $(H, V)$ polarized micro cavity modes. The quantum dot is coupled (coupling constant $g$ ) to both cavity modes due to an orientational mismatch of its dipole (angle $\phi$, see inset).

ventional and unconventional photon blockade effect result in transmitted light with vanishing photon autocorrelation $g^{(2)}(0)<10^{-2}[20,23]$, however, the underlying physical mechanisms are completely different, see Fig. 1. In the strong coupling regime, the unevenly spaced levels of the dressed spectrum prevent reaching the two photon state for a particular laser frequency [red arrows in Fig. 1(a)]. Moreover, the probabilities of having $N>1$ photons in the system are all suppressed with respect to those of a classical state with the same average photon number. In the unconventional photon block- 
ade instead [Fig. 1(b)], only the probability of having $N=2$ photons is suppressed. The sub-poissonian character then arises because, for the chosen pump amplitude, the average photon number - and thus the probabilities of $N>2$ photons - are very small. A possible explanation of the reduced $N=2$ probability is given in terms of the interference between two excitation pathways to the $N=2$ photon state, which can be destructive thanks to the small energy shift of the two-photon state induced by the weak nonlinearity $[17,24]$. An equivalent alternative explanation (discussed below) was proposed in terms of an optimal interplay between squeezing and displacement of the cavity field [25].

We investigate here a single semiconductor quantum dot in an optical micro cavity where a single linearly polarized quantum dot dipole transition is coupled to the two linearly polarized cavity modes due to an orientational mismatch of the quantum dot dipole with respect to the cavity axes [angle $\phi$, see inset Fig. 1(b)]. Since the unconventional photon blockade operates in the low mean photon number regime, Fig. 1(b) shows only the $N=0 \ldots 2$ photon Fock states. Further, we show only one particular excitation pathway (blue), many more involving internal cavity coupling exist but do not qualitatively change the interpretation. More specifically, we rely here on the input- output tuning scheme described in detail in $[19,20]$, here realized via the polarization degree of freedom, which is an extension of the original UPB proposal $[17,18]$. As a result the interference of different excitation pathways with and without involvement of the photonnumber sensitive quantum dot transition can be tuned such that the two- photon component is suppressed.

In this paper, we show experimental evidence of unconventional photon blockade (UPB) in quantum dot cavityQED. The sample consist of a layer of self-assembled InAs/GaAs quantum dots embedded in a micropillar cavity (maximum Purcell factor $F_{p}=11.2$ ) grown by molecular beam epitaxy [26]. The quantum dot layer is embedded in a $\mathrm{P}-\mathrm{I}-\mathrm{N}$ junction, separated by a $27 \mathrm{~nm}$ thick tunnel barrier from the electron reservoir to enable tuning of the quantum dot resonance frequency by the quantum-confined Stark effect. Due to the quantum dot fine-structure structure splitting, we need to consider only one quantum dot transition, which interacts with both the $\mathrm{H}$ and $\mathrm{V}$ cavity modes.

We model our system using a Jaynes-Cummings Hamiltonian in the rotating wave approximation with $g \ll \kappa$. The Hamiltonian for two cavity modes and one quantum dot transition driven by a continuous wave laser is written as

$$
\begin{aligned}
H & =\left(\omega_{L}-\omega_{c}^{V}\right) \hat{a}_{V}^{\dagger} \hat{a}_{V}+\left(\omega_{L}-\omega_{c}^{H}\right) \hat{a}_{H}^{\dagger} \hat{a}_{H} \\
& +\left(\omega_{L}-\omega_{Q D}\right) \hat{\sigma}^{\dagger} \hat{\sigma}+g\left(\hat{\sigma} \hat{b}^{\dagger}+\hat{\sigma}^{\dagger} \hat{b}\right) \\
& +\eta_{H}\left(\hat{a}_{H}+\hat{a}_{H}^{\dagger}\right)+\eta_{V}\left(\hat{a}_{V}+\hat{a}_{V}^{\dagger}\right) .
\end{aligned}
$$

$\omega_{c}^{H}$ and $\omega_{c}^{V}$ are the resonance frequencies of the lin-

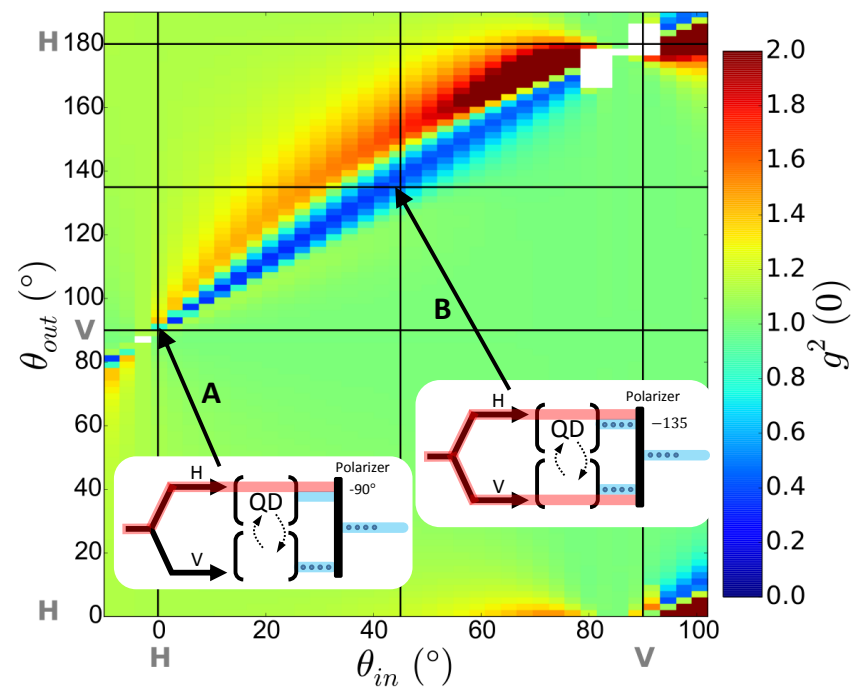

Figure 2. False color plot of the theoretically calculated $g^{(2)}(0)$ convolved with the detector response as a function of the incident and detected linear polarization orientation. Arrow A indicates the condition where most single photon sources operate: the system is excited in the H-cavity mode and the single photons are detected in the V-cavity mode. Arrow $\mathrm{B}$ shows the case where single photons are created using the unconventional photon blockade. White pixels indicate that the simulation has failed due to extremely low photon numbers. The model parameters are given in the supplemental information [29].

early polarized cavity modes, $\hat{a}_{H}^{\dagger}$ and $\hat{a}_{V}^{\dagger}$ the photon creation operators, $\omega_{Q D}$ is the quantum dot resonance frequency, and $\hat{\sigma}^{\dagger}$ the exciton creation operator. $\hat{b}=$ $\hat{a}_{V} \cos \phi+\hat{a}_{H} \sin \phi$ is the cavity photon annihilation operator along the quantum dot dipole orientation, and $\phi$ is the relative angle. In our case the angle is $\phi=94^{\circ}$, which means that the H-cavity mode couples better to the exciton transition. $\eta_{H}$ and $\eta_{V}$ are the amplitudes of the incident coherent light coupling to the $\mathrm{H}$ and $\mathrm{V}$ cavity modes. For numerical simulations, we add relaxation of the cavity modes and dephasing of the quantum dot transition and solve the corresponding quantum master equation $[11,14,27,28]$, add the output polarizer and calculate the mean photon number and second order correlation function. All theoretically obtained $g^{(2)}(\tau)$ data is convolved with the detector response (530 ps) to match the experimental conditions. Details on the device parameters as well as a justification why only a single quantum dot transition has to be taken into account, can be found in the supplemental information [29].

Fig. 2 shows how the second order correlation $g^{(2)}(\tau=0)$ of the transmitted photons depends on the linear input and linear output polarization angle. In all current single photon sources with a quantum dot in a cavity [12-14], only one cavity mode is excited with the laser, and by using a crossed polarizer, single photons are obtained in the orthogonal mode. This condition is indicated with 
arrow A in Fig. 2. By exciting both cavity modes and selecting an appropriate output polarization state such as indicated by arrow B, it is also possible to obtain single photons; this is where the unconventional photon blockade can be observed.

Now, we investigate more closely region B of Fig. 2, where both cavity modes are excited $\left(\theta_{i n}=45^{\circ}\right)$. Furthermore, we add the experimentally unavoidable polarization splitting of the $H$ and $V$ cavity modes which is 10 $\mathrm{GHz}$ for the device under investigation. Furthermore, we vary the detected output polarization in the most general way, by introducing $\lambda / 2$ and $\lambda / 4$ wave plates before the final polarizer in the transmission path. The experimental setup is sketched in the inset of Fig. 3(b) and a more detailed scheme can be found in the supplemental information [29]. Fig. 3(b) shows how this polarization projection affects the mean photon number $\left\langle n_{\text {out }}\right\rangle$, for $\left\langle n_{\text {in }}\right\rangle=\left(\frac{\eta_{H}+\eta_{V}}{\kappa}\right)^{2}=0.06$ in the simulation and in the experiment [Fig. 3(a)]. This region is highly dependent on the cavity splitting and the quantum dot dipole angle, careful determination of the parameters allows us to obtain good agreement to experimental data [Fig. 3(a)]. In this low mean photon number region, the second-order correlation $g^{(2)}(0)$ shows a non-trivial behavior as a function of the output polarization state, shown in Fig. 3(c, experiment) and (d, theory): First, we observe the expected unconventional photon blockade anti-bunching (blue region). The experimentally measured minimum $g^{(2)}(0)$ is $0.37 \pm 0.04$, which is limited by the detector response function. The theoretical data which takes the detector response into account agrees very well to the experimental data and predicts a bare $g^{(2)}(0) \approx 0.005$. Second, we find that, close-by in parameter space, there is a region where bunched photons are produced. This enhancement of the two-photon probability happens via constructive interference leading to phase squeezing. Theoretical and experimental data show good agreement, we attribute the somewhat more extended antibunching region to long-time drifts of the device position during the course of the experiment (10 hours).

In Fig. 3(e) and 3(f) we show the two-time correlation function $g^{(2)}(\tau)$ for the two cases indicated by the arrows. The observed width and height of the anti-bunching and bunching peak predicted by the theory is in agreement with the observed experimental data. The exact shape of the correlation function in Fig. 3(e) is very sensitive to non-ideal effects such as energy fluctuations of the QD, which is why the functional form of the theoretical $g^{(2)}(\tau)$ (blue line Fig. 3) is a bit different compared to the experiment. For two coupled Kerr resonators in the UPB regime, one observes oscillations in $g^{(2)}(\tau)$ when collecting the output of only one of the cavities [18]. During finalizing this paper, a manuscript describing a first observation of this effect has appeared [30]. In our case, these oscillations are absent because the system works mostly as a unidirectional dissipative coupler [31], and the photon field behind the output polarizer contains contributions from both cavities modes, which suppresses
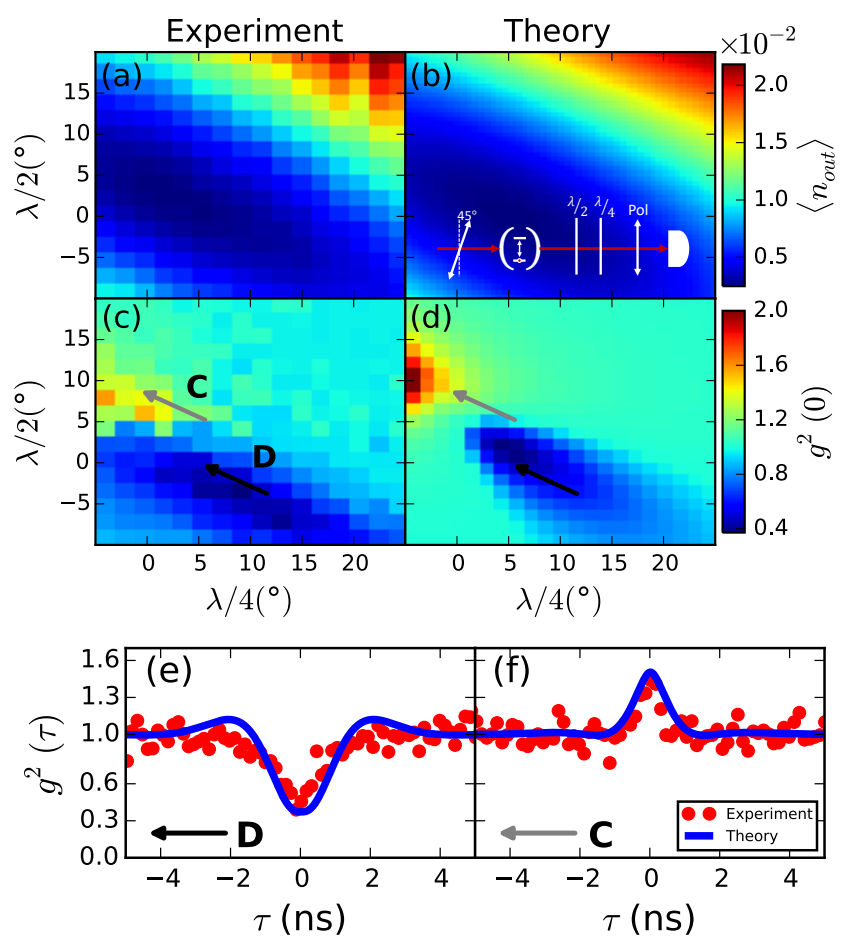

Figure 3. False color plots of $\left\langle n_{\text {out }}\right\rangle$ and $g^{2}(0)$ as a function of the orientation of the $\lambda / 2$ and $\lambda / 4$ wave plate in the transmission path. (a) $\left\langle n_{\text {out }}\right\rangle$ is the mean photon number in a given polarization basis at the output. At $0^{\circ}$ the linear polarized incoming light is parallel to the fast axis of both wave plates. (b) corresponding theory to (a) with as inset a sketch of the experimental setup. (c) and (d) experimental and theoretical $g^{(2)}(0)$. (e) and (f) show $g^{(2)}(\tau)$ for the (anti) bunching region indicated by arrows C (D) in Fig 3(c) and (d). The red dots are measured data and the blue line is the theoretically obtained $g^{(2)}(\tau)$ convolved with the detector response. The exact theoretical parameters are given in the supplemental information [29].

the oscillations in $g^{(2)}(\tau)$.

An alternative way to understand the unconventional photon blockade is in terms of Gaussian squeezed states [25]: For any coherent state $|\alpha\rangle$, there exists an optimal squeeze parameter $\xi$ that minimizes the two-photon correlation $g^{(2)}(0)$, which can be made vanishing for a weak driving fields. We find that, even with a small amount of squeezing, it is possible to significantly reduce the 2 photon distribution and minimize $g^{(2)}(0)$ for low mean photon numbers. A Gaussian squeezed state is produced from vacuum like $D(\alpha) S(\xi)|0\rangle=|\alpha, \xi\rangle$. Here $S$ is the squeeze operator with $\xi=r \exp ^{i \theta}(0 \leq r<\infty, 0 \leq$ $\theta \leq 2 \pi)$. $D$ is the displacement operator, and the complex displacement amplitude $\alpha=\bar{\alpha} \exp ^{i \vartheta}(0 \leq \bar{\alpha}<\infty$, $0 \leq \vartheta \leq 2 \pi)$. For $\theta=\vartheta=0$, we can calculate the two photon probability in the small- $\alpha$ (low mean photon number) limit as

$$
|\langle 2|D(\alpha) S(\xi)| 0\rangle|^{2} \approx\left(\bar{a}^{2}-r\right)^{2} / 2,
$$


(a)

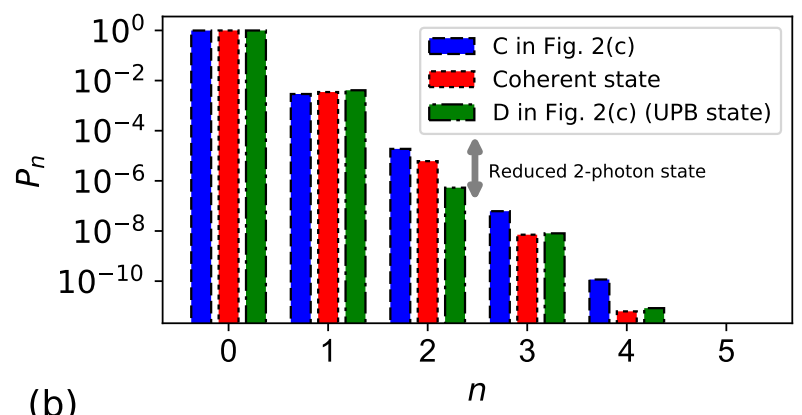

(b)

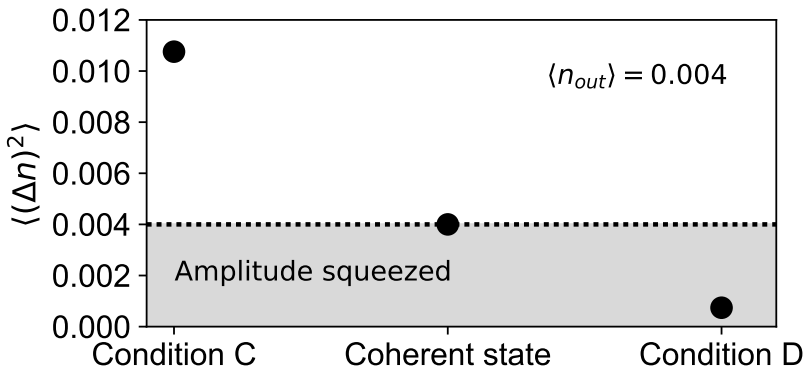

(c)

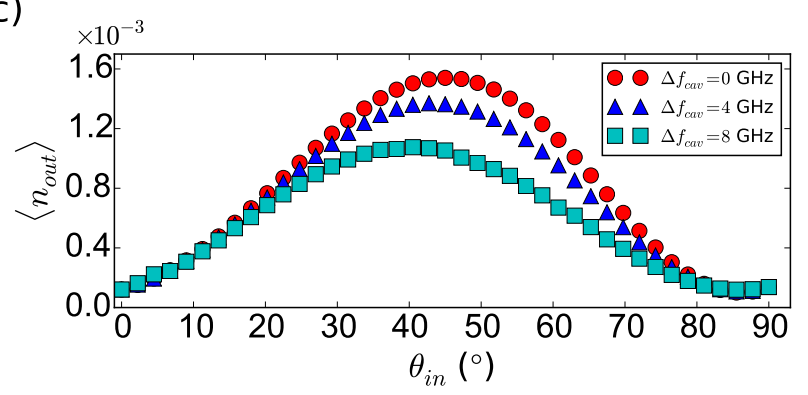

Figure 4. (a) Calculated photon number distribution of a coherent state and for the condition indicated by the arrow $\mathrm{C}$ and $\mathrm{D}$ in Fig. 2(c). (b) The calculated photon number variance for the states presented in (a) showing amplitude squeezing in the region where we observe the unconventional photon blockade. (c) Mean photon number $\left\langle n_{\text {out }}\right\rangle$ as a function of input polarization. We see that a large improvement of the single photon brightness can be obtained by exploiting the UPB effect. The simulation is performed for three cavity splittings $\left(\Delta f_{\text {cav }}\right)$ showing that the enhancement is largest in a polarization degenerate cavity.

using a Taylor expansion. We see that, in order to obtain a vanishing two-photon probability, the squeeze parameter $r$ needs to be equal to $\bar{a}^{2}$ which is the mean photon number. By defining the amount of quadrature squeezing as $\left\langle\left(\Delta X_{1}\right)^{2}\right\rangle=\frac{1}{4} e^{-2 r}$ and considering a $\left\langle n_{\text {out }}\right\rangle \approx 0.004$ (Fig. 3(a)), this condition leads to $10 \log _{10}\left(e^{-0.008}\right)=-3 \times 10^{-2} \mathrm{~dB}$ squeezing. Interestingly, this result means that, for a weak coherent state, only a very small amount of squeezing is needed to make $g^{(2)}(0)$ drop to zero.

In Fig. 4 we show further analysis of the theoretical calculations for the experimental state produced by the un- conventional photon blockade as indicated by arrow $\mathrm{D}$ in Fig. 3(c) and (d). In agreement with equation (1) we observe that the 2-photon state in the photon number distribution shown in Fig. 4(a) is suppressed. By the same mechanism that suppresses the two photon state one also expects to see an increase of the three photon component [19]. The increase of $P_{3}$ is not observed here because of the low mean photon number. From the photon number variance given in Fig. 4(b), we observe that the state is amplitude squeezed. By moving from the region of arrow $\mathrm{C}$ to D in Fig. 3(d), the observed state switches from a phase squeezed to an amplitude squeezed state, which is a clear signature of the unconventional photon blockade effect [19].

Finally, we discuss whether the UPB effect can be used to enhance the performance of single photon sources, and in particular their efficiency. Traditionally, the quantum dot is excited by one linearly polarized cavity mode and photons are collected via the orthogonal mode. In our experiment, the quantum dot excitation probability is $1-\cos \left(4^{\circ}\right) \approx 0.0024$, and, once excited, it has $1-0.0024$ chance to emit into the collection cavity mode, which leads to a low total efficiency. In the unconventional photon blockade regime, arrow B in Fig 2, this efficiency is higher. To further explore this, we show in Fig. 4(c) the mean photon number $\left\langle n_{\text {out }}\right\rangle$ as a function of the input polarization with constant input laser power $\left\langle n_{\text {in }}\right\rangle=0.06$ (the polarization output state is chosen such that $\left.g^{(2)}(0) \approx 0\right)$. We see that, by rotating the input polarization from $0^{\circ}$ to $45^{\circ}$, the output mean photon number can be increased by approximately a factor 10. The simulation is done for various cavity splittings $\Delta f_{\text {cav }}$ which shows that increasing the cavity splitting reduces this enhancement. We conclude that, in the low mean photon number regime, the UPB effect can be used to increase the efficiency a single photon source.

In conclusion, we have experimentally observed the unconventional photon blockade effect using a single quantum dot resonance coupled to two orthogonally polarized cavity modes. We find the expected drop in $g^{(2)}(0)$, but additionally and very close in parameter space, we also find that the transmitted light statistics can be tuned from anti-bunched to bunched, all in good agreement to theoretical models and simulations. In contrast to conventional photon blockade, no energy splitting of the polariton resonances is required, allowing to obtain $g^{(2)}(0) \approx 0$ even with weak non-linearities. Finally, under certain conditions, we find that the unconventional photon blockade effect can increase the efficiency of single photon sources.

\section{ACKNOWLEDGMENTS}

We thank D. Kok and M.F. Stolpe for fruitful discussions. We acknowledge funding from the Netherlands Organisation for Scientific Research (NWO) (Grant No. 
08QIP6-2), from NWO and the Ministry for Education, Culture and Science (OCW) as part of the Frontiers of Nanoscience program, and from the National Science Foundation (NSF) (0901886, 0960331).

[1] Imamoğlu, A., Schmidt, H., Woods, G. \& Deutsch, M. Strongly Interacting Photons in a Nonlinear Cavity. Phys. Rev. Lett. 79, 1467 (1997).

[2] Milburn, G. J. Quantum optical Fredkin gate. Phys. Rev. Lett. 62, 2124 (1989).

[3] Senellart, P., Solomon, G. \& White, A. High-performance semiconductor quantum-dot single-photon sources. Nat. Nanotechnol. 12, 1026 (2017).

[4] Loudon, R. The Quantum Theory of Light (Oxford Science publications) (Oxford University Press, USA, 1973), 3rd edn.

[5] Birnbaum, K. M., Boca, A., Miller, R., Boozer, A. D., Northup, T. E. \& Kimble, H. J. Photon blockade in an optical cavity with one trapped atom. Nature 436, 87 (2005).

[6] Faraon, A., Fushman, I., Englund, D., Stoltz, N., Petroff, P. \& Vučković, J. Coherent generation of non-classical light on a chip via photon-induced tunnelling and blockade. Nat. Phys. 4, 859 (2008).

[7] Lang, C., Bozyigit, D., Eichler, C., Steffen, L., Fink, J. M., Abdumalikov, A. A., Baur, M., Filipp, S., Da Silva, M. P., Blais, A. \& Wallraff, A. Observation of resonant photon blockade at microwave frequencies using correlation function measurements. Phys. Rev. Lett. 106, 243601 (2011).

[8] Hoffman, A. J., Srinivasan, S. J., Schmidt, S., Spietz, L., Aumentado, J., Türeci, H. E. \& Houck, A. A. Dispersive photon blockade in a superconducting circuit. Phys. Rev. Lett. 107, 053602 (2011).

[9] Müller, K., Rundquist, A., Fischer, K. A., Sarmiento, T., Lagoudakis, K. G., Kelaita, Y. A., Sánchez Muñoz, C., Del Valle, E., Laussy, F. P. \& Vučković, J. Coherent generation of nonclassical light on chip via detuned photon blockade. Phys. Rev. Lett. 114, 233601 (2015).

[10] Dousse, A., Lanco, L., Suffczyski, J., Semenova, E., Miard, A., Lematre, A., Sagnes, I., Roblin, C., Bloch, J., Senellart, P., Suffczyński, J., Semenova, E., Miard, A., Lemaître, A., Sagnes, I., Roblin, C., Bloch, J. \& Senellart, P. Controlled Light-Matter Coupling for a Single Quantum Dot Embedded in a Pillar Microcavity Using Far-Field Optical Lithography. Phys. Rev. Lett. 101, 267404 (2008).

[11] Snijders, H., Frey, J. A., Norman, J., Bakker, M. P., Langman, E. C., Gossard, A., Bowers, J. E., Van Exter, M. P., Bouwmeester, D. \& Löffler, W. Purification of a single-photon nonlinearity. Nat. Commun. 7, 12578 (2016).

[12] Somaschi, N., Giesz, V., De Santis, L., Loredo, J. C., Almeida, M. P., Hornecker, G., Portalupi, S. L., Grange, T., Antón, C., Demory, J., Gómez, C., Sagnes, I., Lanzillotti-Kimura, N. D., Lemaítre, A., Auffeves, A., White, A. G., Lanco, L. \& Senellart, P. Near-optimal single-photon sources in the solid state. Nat. Photonics 10, 340 (2016).

[13] Ding, X., He, Y., Duan, Z.-C., Gregersen, N., Chen, M.C., Unsleber, S., Maier, S., Schneider, C., Kamp, M.,
Höfling, S., Lu, C.-Y. \& Pan, J.-W. On-Demand Single Photons with High Extraction Efficiency and Near-Unity Indistinguishability from a Resonantly Driven Quantum Dot in a Micropillar. Phys. Rev. Lett. 116, 020401 (2016).

[14] Snijders, H., Frey, J., Norman, J., Post, V., Gossard, A., Bowers, J., van Exter, M., Löffler, W. \& Bouwmeester, D. Fiber-Coupled Cavity-QED Source of Identical Single Photons. Phys. Rev. Appl. 9, 031002 (2018).

[15] De Santis, L., Antón, C., Reznychenko, B., Somaschi, N., Coppola, G., Senellart, J., Gómez, C., Lemaître, A., Sagnes, I., White, A. G., Lanco, L., Auffèves, A. \& Senellart, P. A solid-state single-photon filter. Nat. Nanotechnol. 12, 663 (2017).

[16] We focus here only on resonantly excited systems.

[17] Bamba, M., Imamoğlu, A., Carusotto, I. \& Ciuti, C. Origin of strong photon antibunching in weakly nonlinear photonic molecules. Phys. Rev. A 83, 021802 (2011).

[18] Liew, T. C. H. \& Savona, V. Single photons from coupled quantum modes. Phys. Rev. Lett. 104, 183601 (2010).

[19] Flayac, H. \& Savona, V. Unconventional Photon Blockade. Phys. Rev. A 96, 053810 (2017).

[20] Flayac, H. \& Savona, V. Input-output theory of the unconventional photon blockade. Phys. Rev. A 88, 033836 (2013).

[21] Gerace, D. \& Savona, V. Unconventional photon blockade in doubly resonant microcavities with second-order nonlinearity. Phys. Rev. A 89, 031803 (2014).

[22] Majumdar, A., Bajcsy, M., Rundquist, A., Vučković, J. \& Vučkovi, J. Loss-Enabled Sub-Poissonian Light Generation in a Bimodal Nanocavity. Phys. Rev. Lett. 108, 183601 (2012).

[23] Verger, A., Ciuti, C. \& Carusotto, I. Polariton quantum blockade in a photonic dot. Phys. Rev. B 73, 193306 (2006).

[24] Wang, C., Liu, Y.-X. Y.-L., Wu, R. \& Liu, Y.-X. Y.L. Phase-modulated photon antibunching in a two-level system coupled to two cavities. Phys. Rev. A 96, 013818 (2017).

[25] Lemonde, M.-A., Didier, N. \& Clerk, A. A. Antibunching and unconventional photon blockade with Gaussian squeezed states. Phys. Rev. A 90, 063824 (2014).

[26] Strauf, S., Stoltz, N. G., Rakher, M. T., Coldren, L. A., Petroff, P. M. \& Bouwmeester, D. High-frequency singlephoton source with polarization control. Nat. Photonics 1, 704 (2007).

[27] Johansson, J., Nation, P. \& Nori, F. QuTiP: An opensource Python framework for the dynamics of open quantum systems. Comput. Phys. Commun. 183, 1760 (2012).

[28] Johansson, J. R., Nation, P. D. \& Nori, F. QuTiP 2: A Python framework for the dynamics of open quantum systems. Comput. Phys. Commun. 184, 1234 (2013).

[29] See Supplemental Material at [URL will be inserted by publisher] for details on the device parameters and experimental setup.

[30] Vaneph, C., Morvan, A., Aiello, G., Féchant, M., Aprili, M., Gabelli, J. \& Estève, J. Observation of the anomalous photon blockade effect in the microwave domain (2018). ArXiv: 1801.04227.

[31] Flayac, H. \& Savona, V. Single photons from dissipation in coupled cavities. Phys. Rev. A 94, 013815 (2016). 